\title{
A scintigraphy study of budesonide/ glycopyrrolate/formoterol fumarate metered dose inhaler in patients with moderate-to-very severe chronic obstructive pulmonary disease
}

\author{
Omar Usmani ${ }^{1 *}$, Nicolas Roche ${ }^{2}$, Ezanul Wahab ${ }^{3}$, Samuel Israel ${ }^{3}$, Martin Jenkins ${ }^{4}$, Roopa Trivedi ${ }^{5}$, \\ Paul Dorinsky ${ }^{5}$ and Magnus Aurivillius ${ }^{6}$
}

\begin{abstract}
Background: Triple therapy with inhaled corticosteroids/long-acting muscarinic antagonists/long-acting $\beta_{2}$-agonists (ICS/ LAMA/LABA) is recommended for patients with chronic obstructive pulmonary disease (COPD) with continued symptoms or exacerbations, despite treatment with LAMA/LABA or ICS/LABA. The pulmonary, extrathoracic, and regional lung deposition patterns of a radiolabeled ICS/LAMA/LABA triple fixed-dose combination budesonide/glycopyrrolate/formoterol fumarate (BGF 320/18/9.6 $\mu$ g), delivered via a single Aerosphere metered dose inhaler (MDI) were previously assessed in healthy volunteers and showed good deposition to the central and peripheral airways (whole lung deposition: 37.7\%). Here, we report the findings assessing BGF in patients with moderate-to-very severe COPD.
\end{abstract}

Methods: This phase I, single-dose, open-label gamma scintigraphy imaging study (NCT03906045) was conducted in patients with moderate-to-very severe COPD. Patients received two actuations of BGF MDI (160/9/4.8 $\mu \mathrm{g}$ per actuation) radiolabeled with technetium-99-pertechnetate, not exceeding $5 \mathrm{MBq}$ per actuation. Immediately following each inhalation, patients performed a breath-hold of up to $10 \mathrm{~s}$, then exhaled into an exhalation filter. Gamma scintigraphy imaging of the anterior and posterior views of the lungs and stomach, and a lateral head and neck view, were performed immediately after exhalation. The primary objective of the study was to assess the pulmonary deposition of BGF. Secondary objectives assessed the deposited dose of radiolabeled BGF in the oropharyngeal and stomach regions, on the actuator, and on the exhalation filter in addition to regional airway deposition patterns in the lungs.

Results: The mean BGF emitted dose deposited in the lungs was 32.1\% (standard deviation [SD] 15.6) in patients with moderate-to-very severe COPD, 35.2\% (SD 12.8) in patients with moderate COPD, and 28.7\% (SD 18.4) in patients with severe/very severe COPD. Overall, the mean normalized outer/inner ratio was 0.55 (SD 0.19), while the standardized central/peripheral ratio was 2.21 (SD 1.64).

\footnotetext{
*Correspondence: o.usmani@imperial.ac.uk

${ }^{1}$ Asthma Lab, National Heart and Lung Institute (NHLI), Imperial College

London \& Royal Brompton Hospital, South Block, Royal Brompton Campus, Sydney St, Chelsea, London SW3 6NP, UK

Full list of author information is available at the end of the article Ezanul Wahab and Samuel Israel: Simbec Research Ltd, Merthyr Tydfil, UK at the time this research was conducted
} original author(s) and the source, provide a link to the Creative Commons licence, and indicate if changes were made. The images or other third party material in this article are included in the article's Creative Commons licence, unless indicated otherwise in a credit line to the material. If material is not included in the article's Creative Commons licence and your intended use is not permitted by statutory regulation or exceeds the permitted use, you will need to obtain permission directly from the copyright holder. To view a copy of this licence, visit http://creativecommons.org/licenses/by/4.0/. The Creative Commons Public Domain Dedication waiver (http://creativeco mmons.org/publicdomain/zero/1.0/) applies to the data made available in this article, unless otherwise stated in a credit line to the data. 
Conclusions: Radiolabeled BGF 320/18/9.6 $\mu \mathrm{g}$ was efficiently delivered and deposited throughout the entire lung, including large and small airways, in patients with moderate-to-very severe COPD, with similar deposition in patients with moderate COPD and patients with severe/very severe COPD.

Trial registration: ClinicalTrials.gov, NCT03906045. Registered 8 April 2019, https://clinicaltrials.gov/ct2/show/NCT03 906045

Keywords: Pulmonary deposition, Gamma scintigraphy, Budesonide, Glycopyrronium, Formoterol fumarate

\section{Introduction}

Patients with chronic obstructive pulmonary disease (COPD) may be prescribed treatment with triple therapy (inhaled corticosteroid/long-acting muscarinic antagonist/ long-acting $\beta_{2}$-agonist [ICS/LAMA/LABA]) if they continue to experience symptoms or exacerbations with dual therapies (LAMA/LABA or ICS/LABA) [1].

Ideally, an inhaled treatment would be consistently deposited in all parts of the lung, including the peripheral airways [2]. Gamma scintigraphy permits the assessment of these regional lung deposition patterns [3-7]. There can be extensive variation in both overall lung deposition and regional deposition patterns of inhaled therapies, dependent on multiple factors, such as the inhalation pattern and delivery device (e.g. metered dose inhaler [MDI] and dry powder inhaler [DPI]), as well as between different devices of the same type. Lung deposition with MDIs may be influenced by the mass of the drug, where a greater mass may reduce the percentage deposited in the lung, in addition to other factors, including the metered volume size and the propellant vapor pressure. Therefore, each MDI product may have unique lung deposition characteristics [8].

The ICS/LAMA/LABA budesonide/glycopyrrolate/ formoterol fumarate (BGF), delivered twice daily via an Aerosphere inhaler (an MDI), is a triple fixed-dose combination therapy that has been approved for the maintenance treatment of COPD in the US, EU, and China, as well as in Japan, to relieve symptoms of COPD [9-12]. BGF was formulated as a suspension with micronized budesonide, micronized glycopyrronium bromide, and micronized formoterol fumarate crystals that were cosuspended with spray-dried porous particles in a hydrofluoroalkane (HFA) propellant (i.e. a single Aerosphere inhaler). Co-suspension delivery technology in the Aerosphere inhaler results in a strong, non-specific association between micronized drug crystals and phospholipidbased porous particles when they are suspended together in the MDI propellant HFA 134a (1,1,1,2-tetrafluoroethane). The resulting suspension is therefore uniform, with consistent dose delivery, even in the presence of simulated patient handling errors, such as a delay between shaking and actuation [13]. The ETHOS study (NCT02465567), which utilized devices with co-suspension delivery technology, showed that BGF significantly reduced moderate or severe COPD exacerbations, and improved lung function and symptoms, compared with corresponding ICS/LABA and LAMA/LABA therapies [14-16]. In addition, significant reductions in risk for all-cause mortality with BGF, compared with the LAMA/LABA glycopyrrolate/formoterol fumarate, were seen in patients with moderate-to-very severe COPD (unadjusted $\mathrm{p}=0.0035$ ) [17].

A previous gamma scintigraphy phase I study evaluated the lung deposition of radiolabeled BGF in healthy male volunteers (NCT03740373) and showed BGF was efficiently deposited in the central and peripheral regions of the lungs, following a 10- and 3 -s breath-hold with an emitted dose in the lungs of $37.7 \%$ and $34.5 \%$, respectively [6]. This study assessed the pulmonary deposition data for radiolabeled BGF, delivered via a single Aerosphere inhaler, in patients with moderate-to-very severe COPD.

\section{Methods}

\section{Study design and treatment}

This was a phase I, single-dose, open-label gamma scintigraphy imaging study (NCT03906045) in patients with moderate-to-very severe COPD (Fig. 1). The screening visit was within 28 days of commencement of the dosing visit and included inhaler training (see below). During the treatment period, patients underwent a Krypton- $81 \mathrm{~m}\left({ }^{81 \mathrm{~m}} \mathrm{Kr}\right)$ gas ventilation scan to define the ventilated area of the lungs. A Cobalt- 57 transmission scan was then performed to evaluate the regional tissue attenuation of deposited radioactivity. BGF was radiolabeled with technetium-99-pertechnetate (no greater than $5 \mathrm{MBq}$ per actuation).

Eligible patients received a single dose of radiolabeled BGF 320/18/9.6 $\mu \mathrm{g}$, consisting of two puffs of 160/9/4.8 $\mu \mathrm{g}$ per actuation, via a single Aerosphere inhaler. There was a breath-hold of up to $10 \mathrm{~s}$ after each inhalation. Gamma scintigraphy imaging was conducted immediately following completion of dosing procedures. Posterior and anterior views of the lungs and stomach, and a lateral head and neck view, were recorded using a gamma camera. Patients were discharged $4 \mathrm{~h}$ post-dose on Day 1 , provided there were no safety concerns. A follow-up phone call was conducted 7-14 days after discharge. 


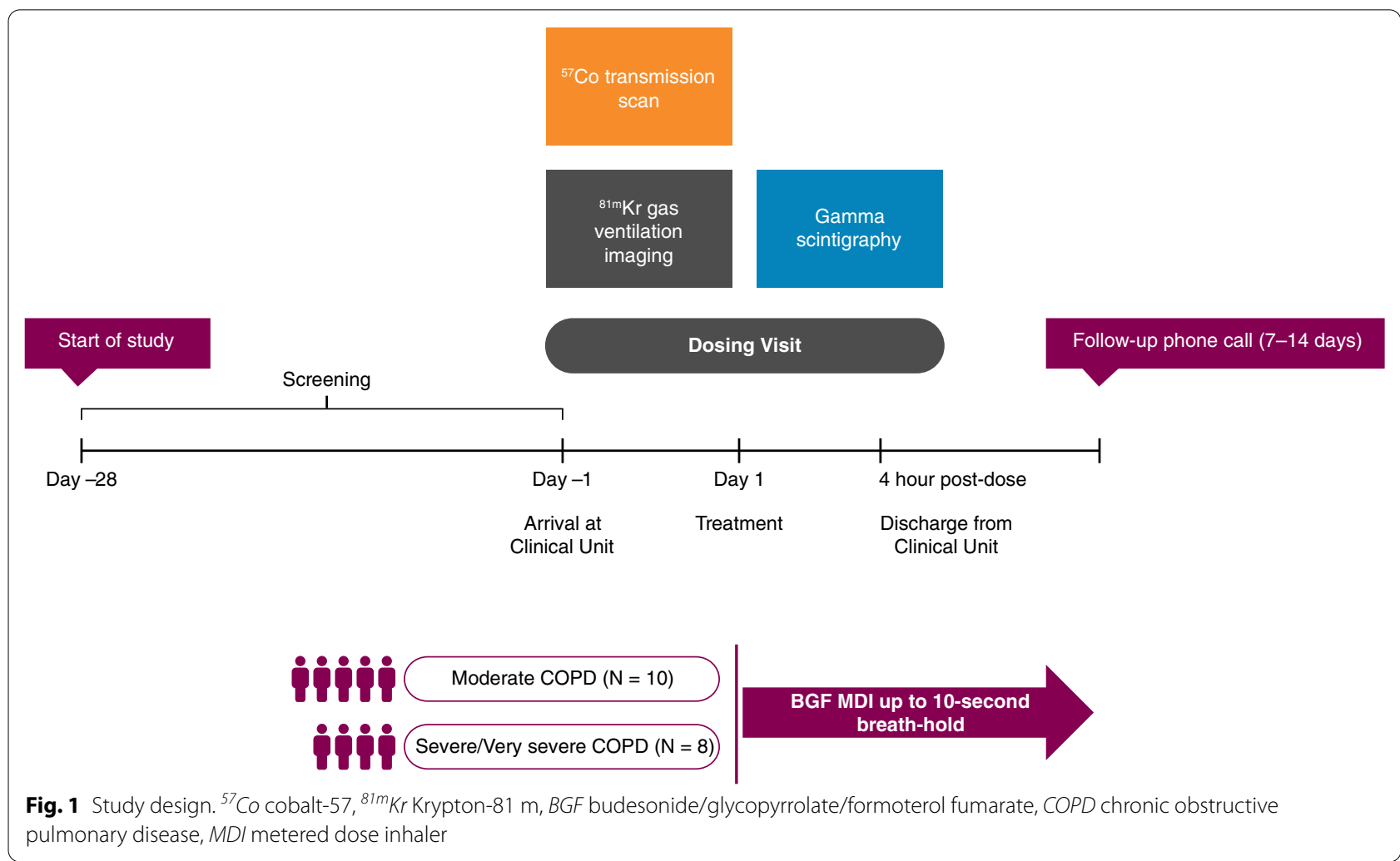

\section{Patients}

\section{Inclusion and exclusion criteria}

At screening and prior to dosing, patients were eligible for participation if they were 40-80 years of age, had a diagnosis of COPD, as defined by the American Thoracic Society/European Respiratory Society, with a post-bronchodilator forced expiratory volume in one second $\left(\mathrm{FEV}_{1}\right)$ /forced vital capacity ratio $<0.70$ and a post-bronchodilator $\mathrm{FEV}_{1}<80 \%$ predicted, were receiving one or more inhaled maintenance therapies (including at least one LAMA or LABA) for management of their COPD for at least four weeks prior to screening, were a current or former smoker with a history of at least 10 pack-years, and were able to demonstrate proper MDI inhalation technique.

Key exclusion criteria were a current diagnosis of asthma, COPD due to an $\alpha_{1}$-antitrypsin deficiency, or any clinically significant respiratory disorder other than COPD. Patients who had experienced a moderate or severe COPD exacerbation within 6 weeks prior to dosing (where the end date of the exacerbation was the last day of treatment with systemic corticosteroids and/or antibiotics) were also excluded.

\section{Inhaler training}

At screening, patients were trained by site staff on the correct use of an MDI using a commercially available MDI training simulator (Vitalograph Aerosol Inhalation Monitor $[\mathrm{AIM}]^{\mathrm{TM}}$; Vitalograph, UK) and an HFA propellant-only MDI. The AIM $^{\mathrm{TM}}$ device was used to confirm that the patient could use the MDI device and stay within the targeted inspiratory flow rate range for the MDI.

On Day -1 (when patients arrived at the clinical unit) and pre-dose on Day 1, patients used a non-radiolabeled propellant-only MDI to confirm they were using the MDI correctly. At any point during the study, site staff re-trained the patient if it was observed that the patient was not using the MDI correctly.

\section{Dose administration}

Patients took two inhalations from the Aerosphere inhaler. Immediately following each inhalation, patients performed a breath-hold of up to $10 \mathrm{~s}$, then exhaled into an exhalation filter. After the second exhalation and breath-hold, patients rinsed their mouth with approximately $20 \mathrm{~mL}$ of water and expelled the washings for 
collection. Patients then swallowed approximately a quarter of a slice of bread and approximately $100 \mathrm{~mL}$ of water to minimize interference from any residual radiolabeled particles in the mouth and throat on the gamma scintigraphy images. Patients continued their regular inhaled maintenance therapies for COPD through Day -1 . On Day 1, regular maintenance therapies were withheld until discharge from the clinical unit and patients were provided ipratropium bromide hydrofluoroalkane (HFA) or albuterol sulfate HFA, which could be used up to, but not within, $6 \mathrm{~h}$ before BGF dosing.

\section{Gamma scintigraphy imaging}

Gamma scintigraphy imaging was conducted immediately following completion of dosing procedures. Posterior and anterior views of the lungs and stomach, and a lateral head and neck view, were recorded using a gamma camera. Gamma scintigraphy images of the MDI were acquired before and after use. Additionally, images of the collected mouth washings and exhalation filter were acquired. All images were obtained over a maximum duration of $200 \mathrm{~s}$.

Prior to the study, the radiolabeling procedures for BGF MDI were validated. In vitro tests were conducted to demonstrate that the aerodynamic particle size distribution of the radiolabel was the same as that for the micronized drug particles, as determined by in vitro Next Generation Impactor (NGI) tests (model 170, Copley Scientific Ltd., United Kingdom). The NGI is a cascade impactor that measures particle size (range of median diameters for model 170: 0.54 to $11.72 \mu \mathrm{m}$ at $30 \mathrm{~L}$ per minute) by moving particles via an air stream that is forced through a series of 7 nozzles with progressively smaller diameters to produce successively higher velocities [18]. Radiolabeled MDIs showed no difference in performance relative to that of the non-radiolabeled control MDIs. On each dosing day prior to treatment administration, in vitro characterization of the radiolabeled MDI product was performed to ensure it complied with predefined release specifications.

\section{Study objectives and outcomes}

The primary objective was to assess the pulmonary deposition of radiolabeled BGF in patients with moderate-tovery severe COPD following a maximal breath-hold of up to $10 \mathrm{~s}$, with deposited doses in the lungs expressed as a percentage of the emitted dose.

Secondary objectives were to assess the deposited dose of radiolabeled BGF in the oropharyngeal and stomach regions, and on the actuator and exhalation filter, in addition to the regional airway deposition patterns in patients with moderate-to-very severe COPD following a maximal breath-hold of up to $10 \mathrm{~s}$.

The deposited doses in the oropharyngeal and stomach regions, and on the exhalation filter, were expressed as a percentage of the emitted dose, and deposition on the actuator was expressed as a percentage of the exvalve (metered) dose. Regional deposition ratios included outer/inner $(\mathrm{O} / \mathrm{I})$ and central/peripheral $(\mathrm{C} / \mathrm{P})$, penetration index (PI; equivalent to the normalized $\mathrm{O} / \mathrm{I}$ ) [19], and standardized $\mathrm{C} / \mathrm{P}(\mathrm{sC} / \mathrm{P})$ ratios [5]. These were calculated for the geometric mean image of the right lung only, as stomach-associated activity may interfere with the left lung image. Regional lung volumes were normalized by comparison to the ${ }^{81 \mathrm{~m}} \mathrm{Kr}$ gas ventilation scan in each patient, allowing for the definition of a rectangle and region of interest to define outer, inner, central, and peripheral areas. $\mathrm{O} / \mathrm{I}$ and $\mathrm{C} / \mathrm{P}$ counts were then obtained for the ${ }^{81 \mathrm{~m}} \mathrm{Kr}$ gas ventilation scan and these values used to account for differences in regional lung volumes. PI represents the regional distribution of the aerosol particles normalized for regional lung volume, and $\mathrm{sC} / \mathrm{P}$ represents an alternative representation of the regional distribution of the aerosol particles normalized for regional lung volume using the ${ }^{81 \mathrm{~m}} \mathrm{Kr}$ gas ventilation scan.

The safety of radiolabeled BGF was assessed based on adverse events (AEs). All AEs were listed and coded using the Medical Dictionary for Regulatory Activities (MedDRA), version 22.0. Serious AEs were recorded from the time of consent to the follow-up phone call, and non-serious AEs were recorded from BGF dosing to follow-up phone call. Only treatment emergent AEs (TEAE) were to be summarized by cohort and overall (both cohorts combined).

\section{Statistical analysis}

Patients were separated into two cohorts, moderate COPD and severe/very severe COPD, to permit an exploratory comparison. Since this was an exploratory comparison, no formal sample size calculation was performed; however, a sample size of at least 16 (eight/ cohort) allowed a direct estimate of lung deposition from this formulation to be made, which is similar to other investigational scintigraphy studies [20, 21].

The primary analysis consisted of descriptive statistics for the primary and secondary endpoints conducted in the per protocol (PP) analysis set, which comprised all patients who received a dose of BGF, had fully evaluable scintigraphy data, and had no protocol violations that may have invalidated or biased the results. A supportive analysis was conducted in patients who received any amount of BGF (safety analysis set). Descriptive statistics were produced for the overall analysis set and within each severity cohort. 
Deposition of BGF in the lungs of patient cohorts with moderate vs. severe/very severe COPD was also assessed. Data in these cohorts were analyzed using an analysis of covariance (ANCOVA) that included COPD severity as a fixed effect, sex as a cofactor, and age, height, and breathhold duration as covariates. Differences in deposition between moderate COPD and severe/very severe COPD were reported as least squares mean (LSM) ( \pm standard error [SE]), along with point estimates and $95 \%$ confidence intervals (CIs).

\section{Results}

\section{Radiolabeling validation}

The NGI analytical tests conducted during radiolabeling method development demonstrated that the aerodynamic size distribution of the emitted aerosol particles for each of the analytes, i.e. budesonide, glycopyrronium, and formoterol fumarate, were comparable to those from the non-radiolabeled canisters, confirming that the radiolabeling process did not alter the performance relative to that of the control nonradiolabeled MDIs. The deposition patterns of each of the analytes are presented in Fig. 2. The effective cutoff diameter $(\mu \mathrm{m})$ for each stage is shown for a flow rate of $30 \mathrm{~L} / \mathrm{min}$, as is the Pharmacopoeial standard [22]. The fine particle fraction for radiolabeled budesonide ranged from $48.69-56.48 \%$ (vs. $46.40-53.51 \%$ for unlabeled), for glycopyrronium ranged from $51.43-59.45 \%$ (vs. $48.74-56.49 \%$ for unlabeled), and for formoterol fumarate ranged from 49.31-56.76\% (vs. $48.03-54.59 \%$ for unlabeled). Tests immediately prior to administration of radiolabeled aerosol on the dosing day also confirmed that each radiolabeled canister delivered the correct amount of radioactivity. Validation of the radiolabeling process confirmed that the radiolabeled aerosols fulfilled criteria described by Devadason and colleagues [3].

\section{Patients}

Of the 48 patients screened, 18 patients were enrolled and dosed (moderate COPD, $\mathrm{N}=10$; severe/very severe COPD, $\mathrm{N}=8$; Fig. 3). Seventeen patients were included in the PP analysis set, since one patient with moderate COPD was excluded due to improper MDI inhalation technique. All patients $(\mathrm{N}=18)$ were included in the safety analysis set.

All enrolled patients were white, 55.6\% were male, with an overall mean age of 62.0 years, and mean $\mathrm{FEV}_{1}$ of $51.1 \%$ predicted. Mean breath-hold time was $9.6 \mathrm{~s}$ (Table 1), with breath-hold duration comparable for patients with moderate and severe/very severe COPD. Of the 8 patients in the severe/very severe COPD cohort, 7 had severe COPD and 1 had very severe COPD.

\section{Lung deposition}

Gamma scintigraphy images for the ${ }^{81 \mathrm{~m}} \mathrm{Kr}$ gas ventilation scan and BGF deposition in representative patients from each cohort are shown in Fig. 4.

The mean percentage of the BGF emitted dose deposited in the lungs was $32.1 \%$ in the overall PP analysis set; $35.2 \%$ for patients with moderate COPD and $28.7 \%$ for patients with severe/very severe COPD (Table 2; Fig. 5).

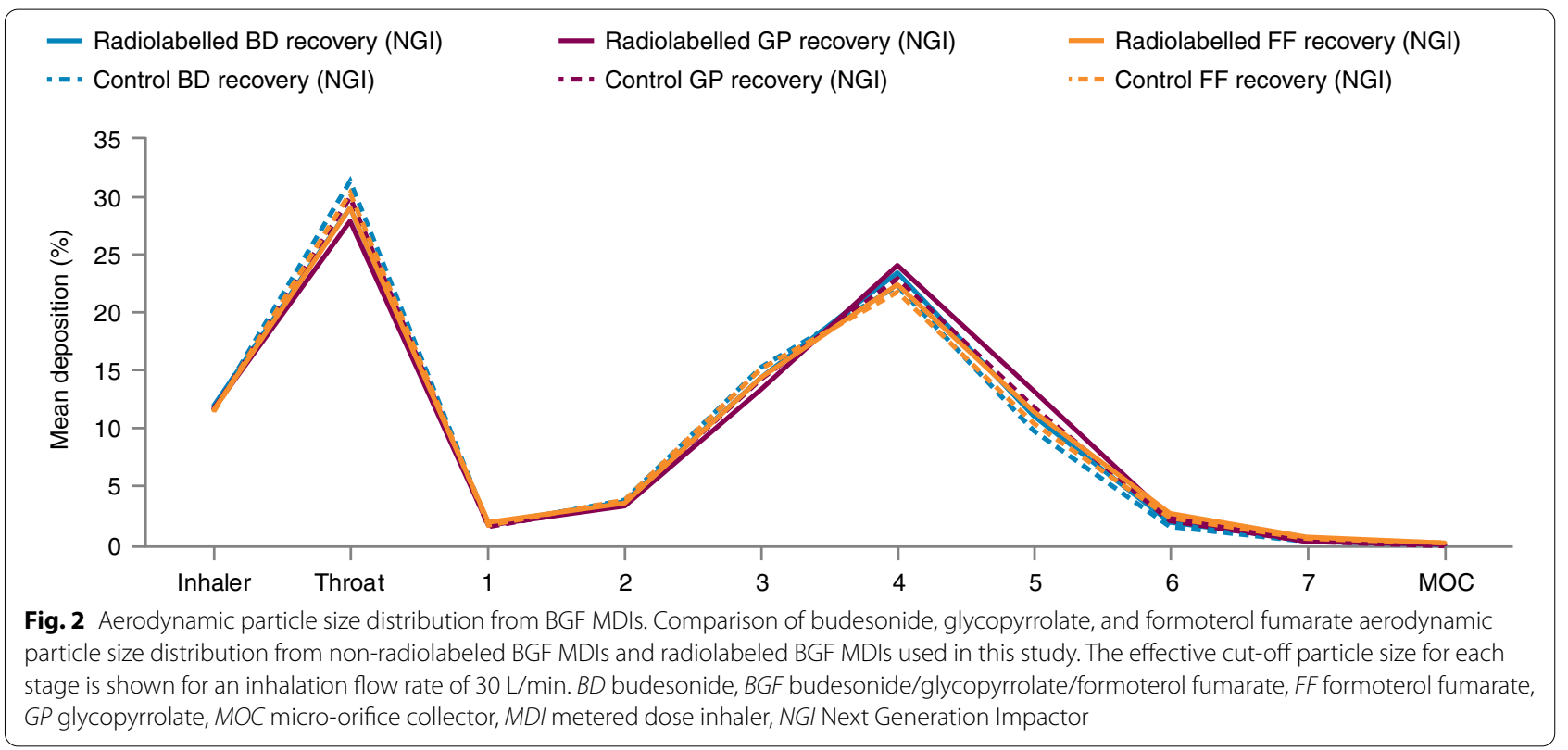




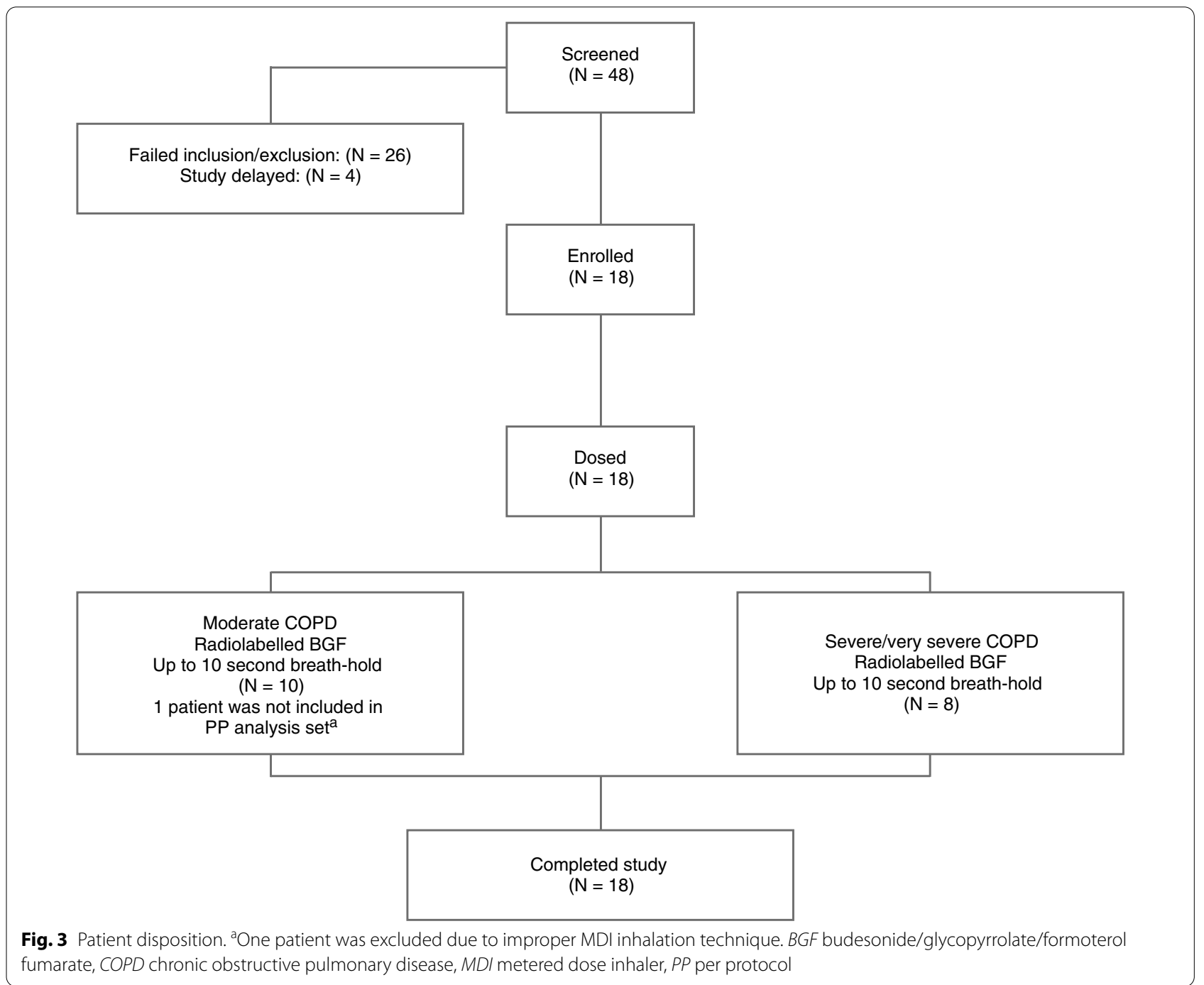

Table 1 Summary of patient demographics and baseline characteristics (safety analysis set)

\begin{tabular}{lccc}
\hline Parameter & Moderate COPD $(\mathbf{N}=10)$ & \multicolumn{1}{c}{ Severe/very severe COPD (N=8) } & Overall (N= 18) \\
\hline Mean age in years (SD) & $61.3(10.8)$ & $62.9(5.7)$ & $62.0(8.7)$ \\
Male, $\mathrm{n}(\%)$ & $6(60.0)$ & $4(50.0)$ & $10(55.6)$ \\
White, $\mathrm{n}(\%)$ & $10(100)$ & $8(100)$ & $18(100)$ \\
Mean BMl, $\mathrm{kg} / \mathrm{m}^{2}$ (SD) & $27.0(3.2)$ & $26.5(4.2)$ & $26.8(3.6)$ \\
Mean years since COPD diagnosis (SD) & $6.6(6.7)$ & $7.8(6.3)$ & $7.1(6.4)$ \\
Mean breath-hold duration, seconds (SD) & $9.5(1.0)$ & $9.7(0.7)$ & $9.6(0.8)$ \\
Mean FEV $\%$ predicted (SD) & $61.1(8.7)$ & $38.6(7.4)$ & $51.1(13.9)$ \\
\hline
\end{tabular}

$B M I$ body mass index, $C O P D$ chronic obstructive pulmonary disease, $F E V_{1}$ forced expiratory volume in one second, $S D$ standard deviation

The estimated difference in BGF emitted dose deposited in the lungs in patients with severe/very severe COPD relative to patients with moderate COPD was minimal (Table 3).
The mean percentage of the BGF emitted dose deposited in the oropharyngeal and stomach regions was $67.2 \%$ in the overall population. A low percentage of the emitted dose was detected in the exhalation filter for radiolabeled 


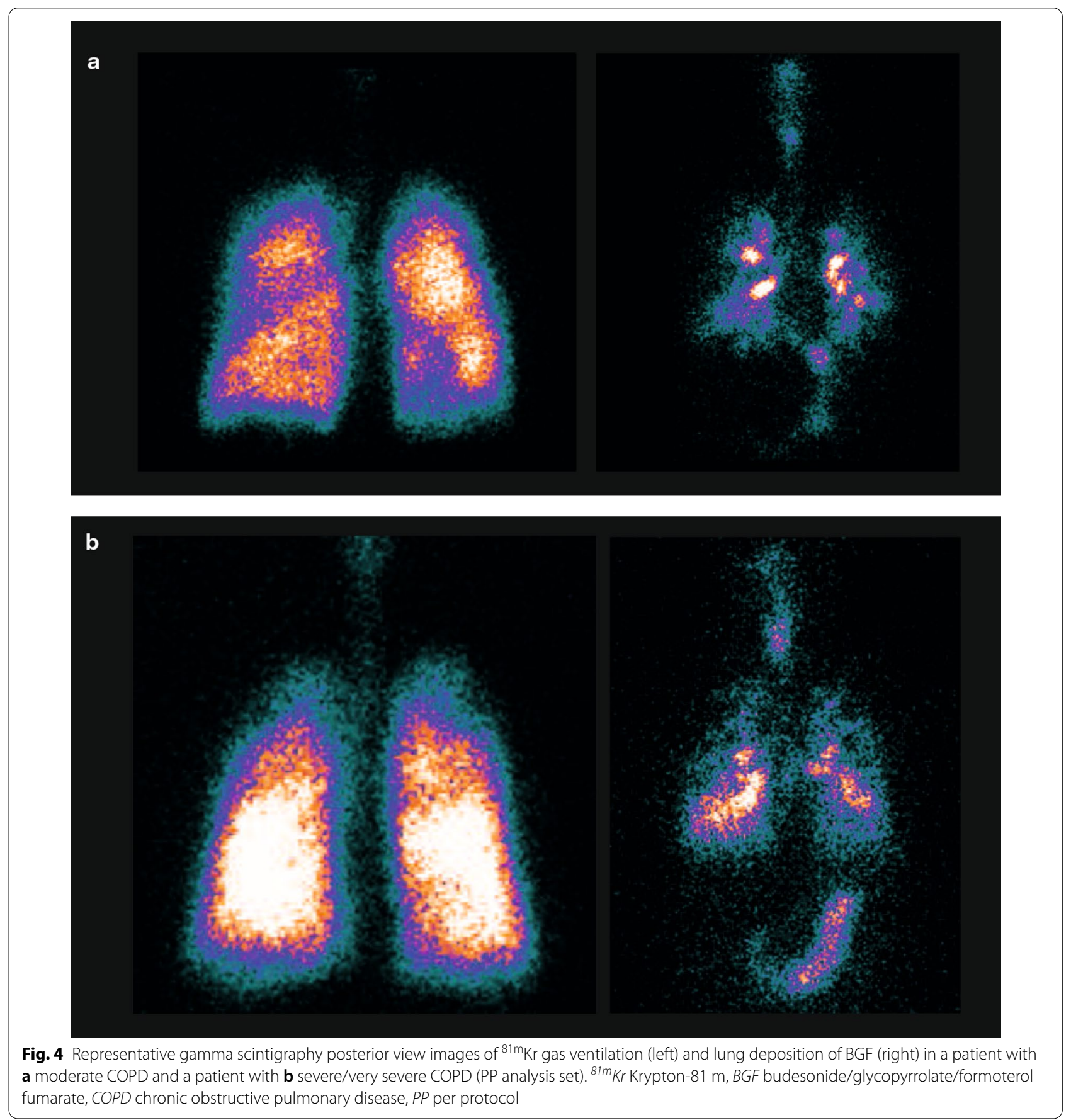

BGF. The mean percentage of radiolabeled BGF detected in the actuator was $11.2 \%$ in the overall population; similar amounts were detected for patients with moderate and severe/very severe COPD (Table 2).

The mean PI regional airway deposition ratio was 0.55 in the overall population, 0.48 in patients with moderate COPD, and 0.63 in patients with severe/very severe COPD. The mean $\mathrm{SC} / \mathrm{P}$ regional airway deposition ratio was 2.21 for the overall population, 2.56 in patients with moderate COPD, and 1.81 in patients with severe/very severe COPD (Table 2). 
Table 2 Summary of derived deposition data ${ }^{a}$ by COPD severity (PP analysis set)

\begin{tabular}{|c|c|c|c|c|c|}
\hline Cohort & Variable & Mean & SD & Median & Min, Max \\
\hline \multirow[t]{9}{*}{ Overall $(N=17)^{b}$} & Emitted dose in lungs, $\%$ & 32.1 & 15.6 & 33.0 & $6.4,52.1$ \\
\hline & Emitted dose in oropharyngeal + stomach regions, $\%$ & 67.2 & 15.1 & 66.2 & $47.5,93.9$ \\
\hline & Emitted dose in exhalation filter, $\%$ & 0.7 & 0.9 & 0.5 & $0.1,3.8$ \\
\hline & Ex-valve dose on actuator, $\%$ & 11.2 & 1.7 & 11.0 & $9.2,15.2$ \\
\hline & Regional airway deposition ratio: & & & & \\
\hline & $\mathrm{O} / \mathrm{l}$ & 1.04 & 0.40 & 1.03 & $0.34,1.95$ \\
\hline & $C / P$ & 0.90 & 0.69 & 0.71 & $0.43,2.94$ \\
\hline & $\mathrm{PI}$ & 0.55 & 0.19 & 0.54 & $0.17,0.89$ \\
\hline & $\mathrm{sC} / \mathrm{P}$ & 2.21 & 1.64 & 1.67 & $1.13,7.19$ \\
\hline \multirow[t]{9}{*}{ Moderate $\operatorname{COPD}(\mathrm{N}=9)$} & Emitted dose in lungs, \% & 35.2 & 12.8 & 41.1 & $9.3,52.1$ \\
\hline & Emitted dose in oropharyngeal + stomach regions, $\%$ & 64.3 & 12.5 & 58.6 & $47.5,89.2$ \\
\hline & Emitted dose in exhalation filter, $\%$ & 0.6 & 0.4 & 0.4 & $0.2,1.5$ \\
\hline & Ex-valve dose on actuator, $\%$ & 12.0 & 1.9 & 11.7 & $9.9,15.2$ \\
\hline & Regional airway deposition ratio: & & & & \\
\hline & $\mathrm{O} / \mathrm{l}$ & 0.93 & 0.31 & 1.03 & $0.34,1.46$ \\
\hline & $\mathrm{C} / \mathrm{P}$ & 1.01 & 0.74 & 0.83 & $0.48,2.94$ \\
\hline & PI & 0.48 & 0.16 & 0.49 & $0.17,0.74$ \\
\hline & $\mathrm{sC} / \mathrm{P}$ & 2.56 & 1.79 & 2.03 & $1.28,7.19$ \\
\hline \multirow{9}{*}{$\begin{array}{l}\text { Severe/very severe COPD } \\
(\mathrm{N}=8)\end{array}$} & Emitted dose in lungs, \% & 28.7 & 18.4 & 25.5 & $6.4,51.5$ \\
\hline & Emitted dose in oropharyngeal + stomach regions, $\%$ & 70.4 & 17.9 & 74.0 & $48.4,92.9$ \\
\hline & Emitted dose in exhalation filter, $\%$ & 0.9 & 1.2 & 0.5 & $0.1,3.8$ \\
\hline & Ex-valve dose on actuator, $\%$ & 10.4 & 1.1 & 10.2 & $9.2,12.7$ \\
\hline & Regional airway deposition ratio: & & & & \\
\hline & $\mathrm{O} / \mathrm{I}$ & 1.17 & 0.48 & 1.20 & $0.40,1.95$ \\
\hline & $C / P$ & 0.78 & 0.66 & 0.54 & $0.43,2.37$ \\
\hline & $\mathrm{PI}$ & 0.63 & 0.20 & 0.68 & $0.27,0.89$ \\
\hline & $\mathrm{sC} / \mathrm{P}$ & 1.81 & 1.45 & 1.35 & $1.13,5.38$ \\
\hline
\end{tabular}

${ }^{\mathrm{a}}$ Following a breath-hold of up to $10 \mathrm{~s}$ after each inhalation

${ }^{b}$ One patient (with moderate COPD) was excluded due to improper MDI inhalation technique

COPD chronic obstructive pulmonary disease, $C / P$ central to peripheral, $M D /$ metered dose inhaler, Min minimum, Max maximum, $O / /$ outer to inner, $P /$ penetration index, $P P$ per protocol, $S C / P$ standardized central/peripheral, $S D$ standard deviation

\section{Safety}

No AEs were reported in this single-dose study.

\section{Discussion}

In patients with moderate-to-very severe COPD, the radiolabeled BGF formulation was efficiently deposited in the lungs, with similar regional deposition patterns for patients with both moderate and severe/very severe COPD. The mean percentage emitted dose in the lungs of patients with moderate COPD was approximately $6 \%$ higher than in patients with severe/very severe COPD, with a corresponding decrease in the mean value deposited in the oropharyngeal and stomach regions (including radioactivity detected in the mouthwash and in the stomach). While the overall magnitude of this difference was small, the fact that some difference was observed is not unexpected given the severity of airway disease in this COPD patient population and the considerable variation in lung deposition values for patients with moderate COPD (9.3-52.1\%) and patients with severe/very severe COPD (6.4-51.5\%). There were no safety concerns identified in this study.

A previous phase I gamma scintigraphy imaging study found that breath-hold duration of 3 vs. $10 \mathrm{~s}$ provided comparable deposition of radiolabeled BGF in healthy male volunteers [6]. Although the study reported herein used a breath-hold of $10 \mathrm{~s}$ (as is generally recommended for MDIs [23]), the methodology for radiolabeling the MDI product, patient training and dose administration, and analysis of gamma scintigraphy images were the same for both studies and therefore allows comparison. The mean percentage of pulmonary deposition for BGF was similar for patients with moderate-to-very severe COPD (32.1\% [SD 15.6]) and for phase I study healthy male volunteers (37.7\% [SD 15.2] [6]). Relative to other MDIs, overall lung deposition with the Aerosphere 


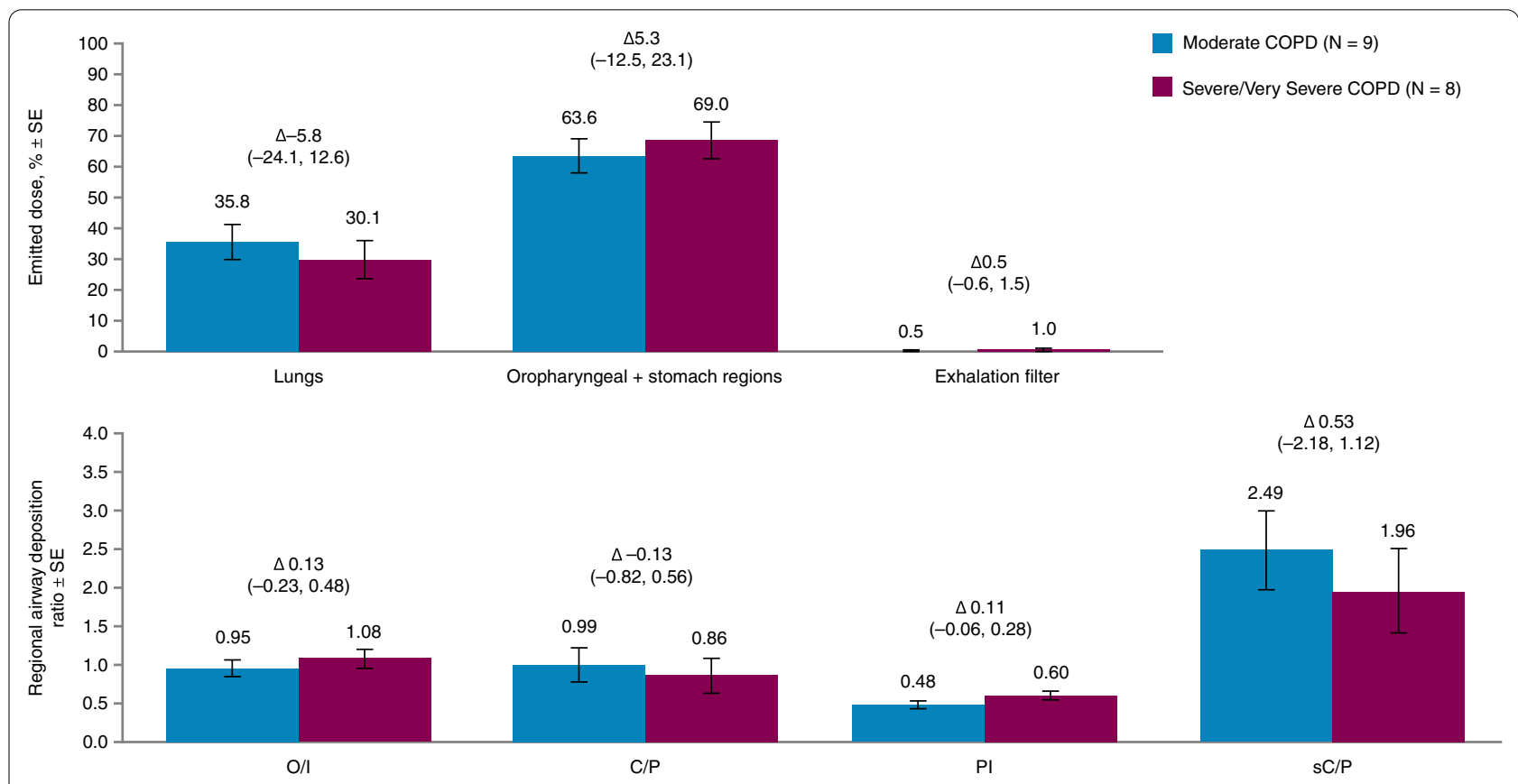

Fig. 5 Derived deposition data for percentage emitted dose and regional deposition ratios in the lung by COPD severity (PP analysis set). $\triangle$ LSM difference for severe/very severe vs. moderate COPD ( $95 \%$ CI), Cl confidence interval, COPD chronic obstructive pulmonary disease, C/P central to peripheral, LSM least squares mean, O/I outer to inner, $P /$ penetration index, $P P$ per protocol, SC/P standardized central/peripheral, SE standard error

Table 3 Lung deposition and regional airway deposition ${ }^{a}$ by COPD severity (PP analysis set)

\begin{tabular}{|c|c|c|c|c|}
\hline \multirow[t]{2}{*}{ Variable } & \multirow{2}{*}{$\begin{array}{l}\text { Moderate COPD } \\
\operatorname{LSM}(\mathrm{SE})(\mathrm{N}=9)\end{array}$} & \multirow{2}{*}{$\begin{array}{l}\text { Severe/very severe COPD } \\
\text { LSM (SE) }(N=8)\end{array}$} & \multicolumn{2}{|c|}{$\begin{array}{l}\text { Severe/very severe vs. moderate } \\
\text { COPD }\end{array}$} \\
\hline & & & $\begin{array}{l}\text { Difference in } \\
\text { LSM (SE) }\end{array}$ & $95 \% \mathrm{Cl}$ \\
\hline Emitted dose in lungs, \% & $35.8(5.7)$ & $30.1(6.1)$ & $-5.8(8.3)$ & $-24.1,12.6$ \\
\hline $\begin{array}{l}\text { Emitted dose in oropharyngeal } \\
+ \text { stomach regions, } \%\end{array}$ & $63.6(5.5)$ & $69.0(5.9)$ & $5.3(8.1)$ & $-12.5,23.1$ \\
\hline Emitted dose in exhalation filter, \% & $0.5(0.3)$ & $1.0(0.3)$ & $0.5(0.5)$ & $-0.6,1.5$ \\
\hline Ex-valve dose on actuator, \% & $12.2(0.6)$ & $10.4(0.6)$ & $-1.8(0.8)$ & $-3.6,0.04$ \\
\hline \multicolumn{5}{|l|}{ Regional airway deposition ratio: } \\
\hline $\mathrm{O} / \mathrm{l}$ & $0.95(0.11)$ & $1.08(0.12)$ & $0.13(0.16)$ & $-0.23,0.48$ \\
\hline $\mathrm{C} / \mathrm{P}$ & $0.99(0.21)$ & $0.86(0.23)$ & $-0.13(0.31)$ & $-0.82,0.56$ \\
\hline PI & $0.48(0.05)$ & $0.60(0.06)$ & $0.11(0.08)$ & $-0.06,0.28$ \\
\hline $\mathrm{sC} / \mathrm{P}$ & $2.49(0.51)$ & $1.96(0.55)$ & $-0.53(0.75)$ & $-2.18,1.12$ \\
\hline
\end{tabular}

${ }^{a}$ Following a breath-hold of up to $10 \mathrm{~s}$ after each inhalation

$C O P D$ chronic obstructive pulmonary disease, $C l$ confidence interval, $C / P$ central to peripheral, $L S M$ least squares mean, $O / /$ outer to inner, $P /$ penetration index, $P P$ per protocol, sC/P standardized central/peripheral, SE standard error

formulation can be considered similar in healthy volunteers and both cohorts of COPD patients [2, 6, 7, 24]. However, in terms of deposition estimates, mean drug deposition declined as lung function worsened, with healthy male volunteers having the highest deposition
(37.7\%) [6], followed by patients with moderate COPD (35.2\%), then patients with severe/very severe COPD (28.7\%). Lung deposition was lower in patients with severe/very severe COPD than in patients with COPD of a lesser severity in this study. However, it should be 
noted that lung deposition with an MDI in patients with severe and very severe COPD who may also have limited inspiratory flow is likely to be greater than would be associated with DPI use. In this regard, an in silico study investigating the influence of inspiratory flow capability and device type on total lung deposition showed that in patients with moderate-to-very severe COPD, use of a DPI was associated with lower total deposition (14-27\%) vs. BGF MDI (40-48\%). More uniform deposition of individual drug components was also observed for MDI vs. DPI use in that study [25]. Since suitable inspiratory flow is a criterion for effective DPI use (the patient must inhale 'hard and fast') [26], for patients with suboptimal inspiratory flow the use of an MDI may be preferable.

As discussed, the overall lung deposition in this study was similar to that described for other HFA dual therapy pressurized MDIs [2, 7] despite the different formulations of other drugs. However, in another study that examined a suspension formulation of fluticasone propionate/salmeterol HFA in patients with asthma, lung deposition was considerably lower (16\%) than we observed in our study of patients with COPD (32.1\%) [27].

For both $\mathrm{O} / \mathrm{I}$ and $\mathrm{C} / \mathrm{P}$, it is important to correct for regional lung volumes by comparison with an ${ }^{81 \mathrm{~m}} \mathrm{Kr}$ gas ventilation scan in each patient. A value close to 1 indicates that the delivered aerosol is deposited throughout the lung airways, equally between large and small airways. Values of $\geq 1$ for PI or $\leq 1$ for $\mathrm{sC} / \mathrm{P}$ indicate a greater proportion in the peripheral airways; conversely, values $<1$ for $\mathrm{PI}$ or $>1$ for $\mathrm{sC} / \mathrm{P}$ indicate a greater proportion in the larger airways. Our data show that administration of BGF resulted in distribution of aerosol particles to all regions of the lungs, and that BGF was delivered to the lungs of patients with COPD with similar efficiency to healthy volunteers (mean PI, 0.55 (SD 0.19) and 0.65 (SD 0.20), respectively) [6]. Moreover, while BGF was distributed in all regions of the lung in both patients with COPD and healthy volunteers, there was a tendency for a somewhat more central deposition in patients with COPD compared to healthy volunteers (mean sC/P, 2.21 (SD 1.64) vs. 1.79 (SD 0.79), respectively) [6].

The low number of patients was a potential limitation of this study, however, the size of the study was similar to that of previous gamma scintigraphy studies $(\mathrm{N}=3-12)[2,6,7,20,21,28-30]$. Another limitation was the wide variation in the lung deposition values in both severity groups (overall: 6.4-52.1\%), despite all patients being carefully trained in the use of an MDI with an AIM inhalation monitor, which is able to visually indicate correct flow rate, co-ordination of actuation and inhalation, and breath-hold. However, this wide variation in lung deposition was also seen in healthy volunteers (20.3-68.3\%) [6]. Although the patient population included patients with severe/very severe airflow, MDI devices pose little or no resistance to airflow and are therefore suited for this population. It is possible that despite training, some patients had a suboptimal MDI inhalation technique. If this is the case, delivery of BGF can be improved with a spacer device [31].

\section{Conclusions}

In conclusion, these results indicate that BGF $320 / 18 / 9.6 \mu \mathrm{g}$ was efficiently deposited throughout the entire lung in patients with moderate-to-very severe COPD. Importantly, deposition was generally similar in patients with moderate COPD and patients with severe/very severe COPD. The results of this study in patients with COPD were generally comparable with BGF deposition observed in prior studies with healthy volunteers, suggesting that despite significant airway disease, BGF is deposited to the entire lung, including the large and small airways.

\section{Abbreviations \\ AAD: Adaptive Aerosol Delivery; AAPS: American Association of Pharma- ceutical Scientists; AE: Adverse event; AIM: Aerosol Inhalation Monitor; BGF: Budesonide/glycopyrrolate/formoterol fumarate; Cl: Confidence interval; COPD: Chronic obstructive pulmonary disease; DPI: Dry powder inhaler; MDI: Metered dose inhaler; NGI: Next Generation Impactor; PI: Penetration index; PP: Per protocol}

\section{Acknowledgements}

This study was supported by AstraZeneca. The authors thank all the patients and the team of investigators involved in this study. The authors thank Cardiff Scintigraphics Ltd, for radiolabeling of the investigational product, gamma scintigraphy imaging, and clinical study report writing support. Medical writing support, under the direction of the authors, was provided by Jake Casson, PhD, CMC Connect, McCann Health Medical Communications, which was funded by AstraZeneca in accordance with Good Publication Practice (GPP3) guidelines [32].

\section{Authors' contributions}

The authors meet criteria for authorship as recommended by the International Committee of Medical Journal Editors, take responsibility for the integrity of the work as a whole, contributed to the writing and reviewing of the manuscript, and have given final approval for the version to be published. All authors had full access to the data in this study and take complete responsibility for the integrity of the data and accuracy of the data analysis. OU, EW, SI, and RT were involved in the acquisition of data and data interpretation. NR, MJ, PD, and MA were involved in conception/design and/or data analysis/ interpretation. All authors read and approved the final manuscript.

Funding

This study was supported by AstraZeneca.

\section{Availability of data and materials}

Data underlying the findings described in this manuscript may be obtained in accordance with AstraZeneca's data sharing policy described at https://astra zenecagrouptrials.pharmacm.com/ST/Submission/Disclosure 


\section{Declarations}

\section{Ethics approval and consent to participate}

Participants completed an informed consent form, approved by the Independent Ethics Committee and sponsor before study initiation. This study was performed in accordance with the ethical principles that have their origin in the Declaration of Helsinki and that are consistent with International Council for Harmonization/Good Clinical Practice, applicable regulatory requirements.

\section{Consent for publication}

Not applicable.

\section{Competing interests}

OU reports academic grants from AstraZeneca, Boehringer Ingelheim, Chiesi, GlaxoSmithKline; and personal fees from Aerocrine, AstraZeneca, Boehringer Ingelheim, Chiesi, Cipla, Mundipharma, NAPP, Prosonix Ltd, Sandoz, Takeda, and Zentiva NR reports grants and personal fees from Boehringer Ingelheim, Novartis, and Pfizer; and personal fees from AstraZeneca, Chiesi, Cipla, GlaxoSmithKline, Mundipharma, Sanofi, Teva, Trudell, and Zambon. EW and SI declare that they have no competing interests. MJ, RT, PD, and MA are employees of, and hold stock and/or stock options in, AstraZeneca.

\section{Author details}

${ }^{1}$ Asthma Lab, National Heart and Lung Institute (NHLI), Imperial College London \& Royal Brompton Hospital, South Block, Royal Brompton Campus, Sydney St, Chelsea, London SW3 6NP, UK. ${ }^{2}$ Respiratory Medicine, Hôpital Cochin (AP-HP), University of Paris, Cochin Institute, Paris, France. ${ }^{3}$ Simbec Research Ltd, Merthyr Tydfil, UK. ${ }^{4}$ AstraZeneca, Cambridge, UK. ${ }^{5}$ AstraZeneca, Durham, NC, USA. ${ }^{6}$ AstraZeneca, Gothenburg, Sweden.

Received: 24 March 2021 Accepted: 28 July 2021

Published online: 07 October 2021

\section{References}

1. Global Initiative for Chronic Obstructive Lung Disease. Global strategy for the diagnosis, management, and prevention of chronic obstructive pulmonary disease 2021 report. 2021. https://goldcopd.org/wp-content/uploads/2020/ 11/GOLD-REPORT-2021-v1.1-25Nov20_WMV.pdf. Accessed 5 Apr 2021.

2. De Backer W, Devolder A, Poli G, Acerbi D, Monno R, Herpich C, et al. Lung deposition of BDP/formoterol HFA pMDI in healthy volunteers, asthmatic, and COPD patients. J Aerosol Med Pulm Drug Deliv. 2010;23(3):137-48.

3. Devadason SG, Chan HK, Haeussermann S, Kietzig C, Kuehl PJ, Newman S, et al. Validation of radiolabeling of drug formulations for aerosol deposition assessment of orally inhaled products. J Aerosol Med Pulm Drug Deliv. 2012;25(Suppl. 1):S6-9.

4. Darquenne C, Fleming JS, Katz I, Martin AR, Schroeter J, Usmani OS, et al. Bridging the gap between science and clinical efficacy: physiology, imaging, and modeling of aerosols in the lung. J Aerosol Med Pulm Drug Deliv. 2016;29(2):107-26.

5. Biddiscombe MF, Meah SN, Underwood SR, Usmani OS. Comparing lung regions of interest in gamma scintigraphy for assessing inhaled therapeutic aerosol deposition. J Aerosol Med Pulm Drug Deliv. 2011;24(3):165-73.

6. Israel S, Kumar A, DeAngelis K, Aurivillius M, Dorinsky P, Roche N, et al. Pulmonary deposition of budesonide/glycopyrronium/formoterol fumarate dihydrate metered dose inhaler formulated using co-suspension delivery technology in healthy male subjects. Eur J Pharm Sci. 2020;153:105472.

7. Taylor G, Warren S, Dwivedi S, Sommerville M, Mello L, Orevillo C, et al. Gamma scintigraphic pulmonary deposition study of glycopyrronium/ formoterol metered dose inhaler formulated using co-suspension delivery technology. Eur J Pharm Sci. 2018;111:450-7.

8. Newman SP. A comparison of lung deposition patterns between different asthma inhalers. J Aerosol Med. 1995;8:S21-6.

9. AstraZeneca Pharmaceuticals LP. Breztri Aerosphere ${ }^{\mathrm{TM}}$ Prescribing Information. 2020. https://www.azpicentral.com/breztri/breztri.pdf. Accessed 2 Feb 2021
10. European Medicines Agency. Trixeo aerosphere. Summary of product characteristics. 2021. https://www.ema.europa.eu/en/documents/produ ct-information/trixeo-aerosphere-epar-product-information_en.pdf. Accessed 2 Feb 2021

11. AstraZeneca. AstraZeneca's triple-combination therapy approved in China for patients with COPD. 2019. https://www.astrazeneca.com/ media-centre/press-releases/2019/astrazenecas-triple-combination-thera py-approved-in-china-for-patients-with-copd-23122019.html. Accessed 2 Feb 2021.

12. AstraZeneca. Breztri Aerosphere (PT010) approved in Japan for patients with chronic obstructive pulmonary disease. 2019. https://www.astra zeneca.com/media-centre/press-releases/2019/breztri-aerospherept010-approved-in-japan-for-patients-with-chronic-obstructive-pulmo nary-disease-19062019.html. Accessed 2 Feb 2021.

13. Doty A, Schroeder J, Vang K, Sommerville M, Taylor M, Flynn B, et al. Drug delivery from an innovative LAMA/LABA co-suspension delivery technology fixed-dose combination MDI: evidence of consistency, robustness, and reliability. AAPS PharmSciTech. 2018;19(2):837-44.

14. Rabe KF, Martinez FJ, Ferguson GT, Wang C, Singh D, Wedzicha JA, et al. Triple inhaled therapy at two glucocorticoid doses in moderate-to-verysevere COPD. N Engl J Med. 2020;383(1):35-48.

15. Rabe KF, Martinez FJ, Singh D, Trivedi R, Jenkins M, Darken P, et al. Improvements in lung function with budesonide/glycopyrrolate/ formoterol fumarate metered dose inhaler versus dual therapies in patients with COPD: a sub-study of the ETHOS trial. Ther Adv Respir Dis. 2021;15:17534666211034329. https://doi.org/10.1177/17534666211034329.

16. Martinez FJ, Rabe KF, Ferguson GT, Wedzicha JA, Trivedi R, Jenkins M, et al. Benefits of budesonide/glycopyrrolate/formoterol fumarate (BGF) on symptoms and quality of life in patients with COPD in the ETHOS trial Respir Med. 2021;185:106509. https://doi.org/10.1016/j.rmed.2021.106509.

17. Martinez FJ, Rabe KF, Ferguson GT, Wedzicha JA, Singh D, Wang C, et al. Reduced all-cause mortality in the ETHOS trial of budesonide/glycopyrrolate/formoterol for chronic obstructive pulmonary disease. A randomized, double-blind, multicenter, parallel-group study. Am J Respir Crit Care Med. 2021:203(5):553-64.

18. van Geffen GJ, Markerink H, van Barneveld M, Verhoeven F, Scheffer GJ, Bruhn J. Comparative in-vitro study of the trachospray, a new device for topical anaesthesia of the upper airway. Med Devices (Auckl). 2021;14:9-14.

19. Newman S, Bennett WD, Biddiscombe M, Devadason SG, Dolovich MB, Fleming J, et al. Standardization of techniques for using planar (2D) imaging for aerosol deposition assessment of orally inhaled products. J Aerosol Med Pulm Drug Deliv. 2012:25(Suppl. 1):S10-28.

20. Sebti T, Pilcer G, Van Gansbeke B, Goldman S, Michils A, Vanderbist F, et al. Pharmacoscintigraphic evaluation of lipid dry powder budesonide formulations for inhalation. Eur J Pharm Biopharm. 2006;64(1):26-32.

21. Shrewsbury SB, Armer TA, Newman SP, Pitcairn G. Breath-synchronized plume-control inhaler for pulmonary delivery of fluticasone propionate. Int J Pharm. 2008;356(1-2):137-43.

22. United States Pharmacopeial Convention Inc. [601] Inhalation and nasal drug products: aerosols, sprays, and powders-n performance quality tests. C.7 Apparatus 6 for inhalation aerosol and sprays - next generation impactor (without pre-separator). In: 2017 US Pharmacopoeia-national formulary [USP 40 NF 35]. Rockville, MD; 2017:498. http://www.uspbpep. com/usp31/v31261/usp31nf26s1_c601.asp. Accessed 30 Aug 2021.

23. Pleasants RA, Hess DR. Aerosol delivery devices for obstructive lung diseases. Respir Care. 2018;63(6):708-33.

24. Leach CL, KuehI PJ, Chand R, McDonald JD. Respiratory tract deposition of HFA-beclomethasone and HFA-fluticasone in asthmatic patients. J Aerosol Med Pulm Drug Deliv. 2016;29(2):127-33.

25. Wu L, Van Holsbeke C, Mack P. Consistent lung delivery of inhaled triple ICS/LAMA/LABA combination using the co-suspension delivery technology: an in silico modelling study. San Antonio: American Association of Pharmaceutical Scientists (AAPS); 2019.

26. Mahler DA. Peak inspiratory flow rate as a criterion for dry powder inhaler use in chronic obstructive pulmonary disease. Ann Am Thorac Soc. 2017:14(7):1103-7.

27. Leach CL, Kuehl PJ, Chand R, Ketai L, Norenberg JP, McDonald JD. Characterization of respiratory deposition of fluticasone-salmeterol 
hydrofluoroalkane-134a and hydrofluoroalkane-134a beclomethasone in asthmatic patients. Ann Allergy Asthma Immunol. 2012;108(3):195-200.

28. Hirst PH, Pitcairn GR, Weers JG, Tarara TE, Clark AR, Dellamary LA, et al. In vivo lung deposition of hollow porous particles from a pressurized metered dose inhaler. Pharm Res. 2002;19(3):258-64.

29. Nikander K, Prince I, Coughlin S, Warren S, Taylor G. Mode of breathingtidal or slow and deep-through the I-neb Adaptive Aerosol Delivery (AAD) system affects lung deposition of 99mTc-DTPA. J Aerosol Med Pulm Drug Deliv. 2010;23(Suppl. 1):S37-43.

30. Weers J, Metzheiser B, Taylor G, Warren S, Meers P, Perkins WR. A gamma scintigraphy study to investigate lung deposition and clearance of inhaled amikacin-loaded liposomes in healthy male volunteers. J Aerosol Med Pulm Drug Deliv. 2009;22(2):131-8.
31. Dorinsky P, DePetrillo P, DeAngelis K, Trivedi R, Darken P, Gillen M. Relative bioavailability of budesonide/glycopyrrolate/formoterol fumarate metered dose linhaler administered with and without a spacer: results of a phase I, randomized, crossover trial in healthy adults. Clin Ther. 2020;42(4):634-48.

32. Battisti WP, Wager E, Baltzer L, Bridges D, Cairns A, Carswell Cl, et al. Good publication practice for communicating company-sponsored medical research: GPP3. Ann Intern Med. 2015;163(6):461-4.

\section{Publisher's Note}

Springer Nature remains neutral with regard to jurisdictional claims in published maps and institutional affiliations.
Ready to submit your research? Choose BMC and benefit from:

- fast, convenient online submission

- thorough peer review by experienced researchers in your field

- rapid publication on acceptance

- support for research data, including large and complex data types

- gold Open Access which fosters wider collaboration and increased citations

- maximum visibility for your research: over 100M website views per year

At BMC, research is always in progress.

Learn more biomedcentral.com/submissions 